Check for updates

Cite this: RSC Adv., 2019, 9, 24357

\title{
Facile preparation of superhydrophobic wood surfaces via spraying of aqueous alkyl ketene dimer dispersions
}

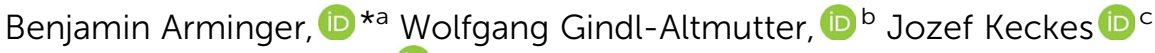 \\ and Christian Hansmann (1D)
}

\begin{abstract}
The prevention of excessive water uptake in wood in order to avert discoloration, swelling and decay is a major challenge for wood-based applications. We developed a facile surface treatment to protect wood from liquid water uptake that does not require harsh process conditions or toxic solvents. Waterbased and surfactant-free dispersions of sub-micron alkyl ketene dimer wax particles were prepared and sprayed onto wood substrates. After the evaporation of water, the wax particles self-assembled into distinctive platelet structures. Depending on the specific conditions of application, water contact angles as high as $166^{\circ}$ were measured on treated wood surfaces. The implementation of sub-micro structures clearly reduced surface gloss but transparency and color remained largely unaffected. The method is comparably cost-effective and scalable, overcoming dimensional limitations crucial for many applications of wood
\end{abstract}

Received 16th May 2019

Accepted 31st July 2019

DOI: $10.1039 / c 9 r a 03700 d$

rsc.li/rsc-advances

approaches that target optimized bulk behavior during water

\section{Introduction}

Wood is a renewable, easily accessible and inexpensive resource that has been used by humankind for thousands of years. As a building or furniture material it has numerous favorable properties such as high stiffness and strength at comparably low weight, ${ }^{1}$ appealing visual appearance, and low embodied energy and carbon impact. ${ }^{2}$ Today, due to the growing awareness for the environmental impact of different materials, but also because of demand for its advantageous properties, the use of wood is increasing. ${ }^{3}$ Major drawbacks of wood as a structural material lie in its susceptibility to biological degradation, and in dimensional instabilities that can result in cracking or warping. Both can reduce the service life of wood-derived products dramatically. These issues are caused by fundamentally different processes but share one common origin, i.e. the water uptake of wood due to its hydrophilic nature.

During the past decades, extensive research was carried out on measures preventing decay and dimensional instabilities of wood. Conventional wood modification comprises various

${ }^{a}$ Wood K plus - Competence Centre for Wood Composites and Wood Chemistry, Konrad-Lorenz-Straße 24, 3430 Tulln, Austria. E-mail: b.arminger@wood-kplus.at; c.hansmann@wood-kplus.at

${ }^{b}$ Department of Material Science and Process Engineering, Institute of Wood Technology and Renewable Materials, BOKU University of Natural Resources and Life Sciences, Konrad-Lorenz-Straße 24, 3430 Tulln, Austria. E-mail: wolfgang. gindl@boku.ac.at

'Department of Materials Physics, Erich Schmid Institute of Materials Science, Montanuniversität Leoben, Jahnstraße 12/I, 8700 Leoben, Austria. E-mail: jozef. keckes@unileoben.ac.at contact. ${ }^{4-6}$ Thermal and chemical modification - essentially acetylation and furfurylation - turned out to be the most promising methods and resulted in successful market implementations. ${ }^{7}$

Nevertheless, bulk wood modification faces fundamental drawbacks such as the necessity for elaborate processes, unsuitability of certain wood species, or dependence on high chemical loadings. Often, modification results in reduced mechanical properties or unwanted color changes. Such disadvantages might increase the prices or limit possible applications of modified wood products. To overcome the wetting of wood with water and ensuing dimensional change or biodegradation, recent research aimed for the development of highly water-repelling surfaces. This approach follows an interdisciplinary trend of superhydrophobicity research that started in the beginning of this century and has now reached most diverse industries and materials. ${ }^{8}$ Superhydrophobic surfaces are characterized by water contact angles (WCA) higher than $150^{\circ}$ and can show other interesting features such as self-cleaning, anti-icing or anti-fogging ability. ${ }^{9,10}$ Initiated by insight on the surface of the Lotus (Nelumbo nucifera) leaf, ${ }^{11}$ theoretical principles for such exceptional water-repellency were discussed frequently, ${ }^{12,13}$ focusing on hierarchical structures created by low surface free energy materials. The first approaches for the preparation of superhydrophobic wood surfaces were based on the deposition of zinc oxide onto the wood cell wall, which was then modified with stearic acid. ${ }^{\mathbf{1 4 1} 15}$ The natural roughness of wood provides a primary surface 
pattern on the microscale. Implementation of a second, nanoscale roughness can then lead to superhydrophobic hierarchical surface structures. Following this principle, several approaches were based on the deposition and subsequent modification of inorganic nanostructures such as silica, ${ }^{16-18} \mathrm{TiO}_{2},{ }^{19,20} \mathrm{WO}_{3},{ }^{21}$ or $\mathrm{ZnO}^{22}$ Others applied small-scale structure onto a hydrophobic PDMS coating. ${ }^{23-25}$ These modified surfaces of intrinsically hydrophilic wood substrate reached WCA above $150^{\circ}$ but were prepared using complex or time-consuming processes. Most of these procedures consist of two or more production steps, including at least one immersion or autoclave treatment hardly suitable for continuous and efficient large scale production. Few single-step applications have been reported. Examples are immersion in PMS solution for 18 hours, ${ }^{26}$ or a six-hour hydrothermal treatment in an autoclave, ${ }^{27}$ resulting in similar drawbacks as mentioned above. Further, many of the above-named approaches are based on the use of hazardous raw materials or solvents. In contrast, a more environmentally-friendly process was published by Lozhechnikova et al. ${ }^{28}$ who developed surfactant-free carnauba wax dispersions for layer-by-layer deposition with ZnO nanoparticles on wood surfaces. Still, 8 bilayers had to be developed via 16 immersion repetitions to reach superhydrophobicity.

Similarly to wood, also paper requires a certain degree of water-repellency in many applications, which is why decades of experience in wax-based hydrophobisation of cellulosic material can be found in paper industry. Alkyl ketene dimer (AKD) emulsion patented in $1949^{29}$ is the most widely used paper sizing agent beside alkenyl succinic anhydride. ${ }^{30,31}$ AKD consists of a 2-oxetanone ring system with an alkyl group and an alkylidene group attached on the 3- and 4-position, respectively (Fig. 1). For fully AKD sized paper, maximum (advancing) WCA of $110^{\circ}$ are reported. ${ }^{32}$ Significantly higher WCA has been shown for AKD-coated glass substrate ${ }^{33,34}$ where the drying of an AKD melt under

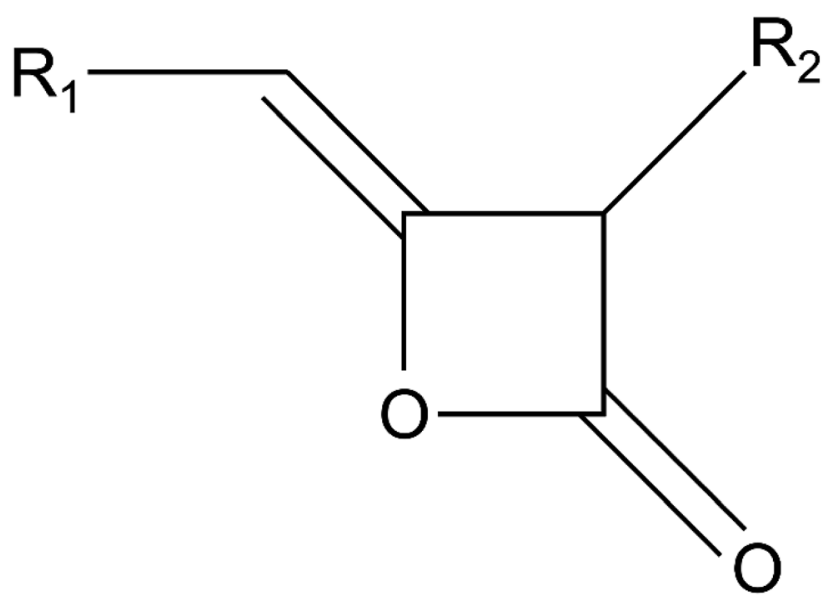

Fig. 1 Chemical structure of alkyl ketene dimers with $R_{1}$ and $R_{2}$ representing straight carbon chains of usually $16-18$ carbon atoms in length. nitrogen gas atmosphere resulted in the growth of fractal structures exhibiting WCA as high as $174^{\circ}$. Later, a superhydrophobic AKD coating of similar structure was developed on paper using rapid expansion of supercritical $\mathrm{CO}_{2} \cdot{ }^{35}$ High pre-expansion pressure from 100-300 bar but no toxic solvents were used in this process. The resulting surface consisted of thin, randomly oriented but mainly upright AKD platelets of few micrometers in length.

The present study reports the utilization of AKD for generating highly water repellant wood surfaces. Based on the spraying of water-based dispersions of sub-micron AKD particles, we aim at a simple and straightforward process involving neither high temperature or pressure, nor sophisticated infrastructure or the extensive use of solvents. The influence of particle size, the number of spraying repetitions, and the drying conditions on the ultimate wetting behavior will be investigated together with the effect of AKD coating on the visual appearance of wood surfaces.

\section{Materials and methods}

\section{Materials}

AKD FennoWax C-18EX was provided by Kemira Chemie GesmbH (Krems, Austria) and used without further purification. European beech (Fagus sylvatica) wood used as a substrate was cut into samples of $30 \mathrm{~mm} \times 30 \mathrm{~mm} \times 5 \mathrm{~mm}$ in radial $\times$ longitudinal $\times$ tangential direction, respectively. The samples were sanded using P280 sandpaper, washed and rinsed with acetone, and dried at room temperature.

\section{Preparation and characterization of aqueous AKD dispersions}

Differential scanning calorimetry of AKD was performed using a DSC 200 F3 Maia (Netzsch-Gerätebau GmbH, Selb, Germany) at a scan rate of $10 \mathrm{~K} \mathrm{~min}^{-1}$. It revealed a melting range of $53-72{ }^{\circ} \mathrm{C}$. To obtain AKD particles, the wax was melted in water at $80{ }^{\circ} \mathrm{C}$, emulsified in liquid state, and cooled down for re-solidification. In total, eight different dispersions with target AKD concentrations of 1 or $10 \mathrm{~g} \mathrm{~L}^{-1}$ water were prepared applying the following methods (Table 1). For the preparation of the dispersions I-IV, the melted AKD was emulsified by 7 min of blending using a KSB-200 (KeMar GmbH, Munich, Germany) blender. Dispersions VVIII were emulsified by 2 min of blending followed by $5 \mathrm{~min}$ of ultrasonication using a Q500 CL-334 sonicator (Qsonica L.L.C, Newtown, USA) with $1 / 2^{\prime \prime}$ probe diameter. Preliminary experiments have revealed high repeatability when the particles re-solidified during exposure to low or high - but not moderate - temperature gradients. Thus, after emulsification, the specimens were either kept in beakers until they reached room temperature, or poured into a metal pot set in ice water for rapid cooling. All dispersions were finally filtered through a stainless steel filter of $35 \mu \mathrm{m}$ nominal pore size.

The resulting mean particle size and polydispersity index (PDI) within each dispersion were measured by means of dynamic light scattering (DLS) using a Zetasizer Nano ZS 
Table 1 Preparation methods of AKD dispersions and their particle properties ${ }^{a}$

\begin{tabular}{|c|c|c|c|c|c|c|c|c|}
\hline $\begin{array}{l}\text { Dispersion } \\
\text { I }\end{array}$ & \multicolumn{4}{|l|}{ Preparation } & \multicolumn{4}{|c|}{ Particle properties } \\
\hline II & 1 & 7 & 0 & Ice & 340 & \pm 4 & 0.29 & \pm 0.01 \\
\hline III & 10 & 7 & 0 & $\mathrm{RT}$ & 1.133 & \pm 27 & 0.56 & \pm 0.03 \\
\hline IV & 10 & 7 & $\mathbf{0}$ & Ice & 841 & $\pm \mathbf{4 0}$ & 0.44 & $\pm \mathbf{0 . 0 4}$ \\
\hline VII & 10 & 2 & 5 & RT & 291 & \pm 7 & 0.42 & $\pm \mathbf{0 . 0 2}$ \\
\hline VIII & 10 & 2 & 5 & Ice & 298 & \pm 2 & 0.44 & \pm 0.01 \\
\hline
\end{tabular}

${ }^{a}$ TWC target wax concentration, BT blending time, UST ultrasonication time, RT room temperature, PDI polydispersity index. Errors for particle properties represent standard deviations.

(Malvern Panalytical GmbH, Kassel, Germany). Two measurements on duplicate samples of each dispersion were carried out. The electrokinetic potential was measured 9 days after the preparation using the same Zetasizer Nano ZS instrument. For further information on particle size and shape, atomic force microscopy (AFM) was carried out using a Dimension Icon Scanning Probe Microscope (Nanoscope V, Bruker Corporation, Billerica, USA) in ScanAsyst ${ }^{\circledR}$ mode at a scan rate of $0.1 \mathrm{~Hz}$. Particles from highly diluted dispersions dried on mica surfaces were scanned in air with ScanAsyst-Air tips (Bruker) with a nominal resonant frequency of $70 \mathrm{kHz}$ and a nominal spring constant of $0.4 \mathrm{~N} \mathrm{~m}^{-1}$.

\section{Spray coating and drying}

Dispersions were sprayed on wood surfaces using an Evolution (Harder \& Steenbeck GmbH \& Co. KG, Norderstedt, Germany) airbrush tool equipped with a $0.15 \mathrm{~mm}$ nozzle and the corresponding needle. Consequently, water was evaporated from the wood surface at temperatures ranging from $20-80{ }^{\circ} \mathrm{C}$ before the spraying-drying procedure was repeated. To investigate the influence of the number of repetitions, samples were coated 212 times and intermittently dried at $40{ }^{\circ} \mathrm{C}$ for $10 \mathrm{~min}$. For the evaluation of potential effects of drying temperature, samples were coated 4 times and dried for $5 \mathrm{~min}$ at $20-80{ }^{\circ} \mathrm{C}$ after each spraying. To investigate the influence of extended exposure to temperature, one of these samples was kept in the oven at $40{ }^{\circ} \mathrm{C}$ for $24 \mathrm{~h}$.

\section{Characterization of coated wood surfaces}

The wood surface color parameters $L^{*}, a^{*}$, and $b^{*}$ within the CIELab system were measured with a Spectrophotometer PCECSM 8 (PCE Instruments, Meschede, Germany) on the same position before and after the coating was applied. The color change expressed using the CIELab parameter for color distance $\left(\Delta E^{*}\right)$ was calculated using formula (1), where $L^{*}$ is the lightness value, $a^{*}$ represents the green-red and $b^{*}$ the blueyellow color components. Similarly, gloss measurements were performed on the samples before and after the spray treatments. Values at $20^{\circ}, 60^{\circ}$, and $85^{\circ}$ measurement angle were generated using a micro-TRI-gloss tool (BYK-Gardner $\mathrm{GmbH}$,
Geretsried, Germany). According to the DIN EN ISO 2813:201502 standard, the values at $85^{\circ}$ angle were used for gloss change calculations.

$$
\Delta E^{*}=\sqrt{\left(\Delta L^{*}\right)^{2}+\left(\Delta a^{*}\right)^{2}+\left(\Delta b^{*}\right)^{2}}
$$

The static WCA of sessile drops was measured for $60 \mathrm{~s}$ using a semiautomatic Drop Shape Analyzer - DSA 30S (Krüss GmbH, Hamburg, Germany). Two water droplets of $10 \mu \mathrm{L}$ volume were placed on duplicate specimens for each type of coating. For a period of $1 \mathrm{~min}$ after placing the droplet onto the surface, 10 images per second were recorded with a digital camera. Drop shape was analyzed by means of built-in image analysis software and contact angles were calculated using the Young-Laplace fit. It models a drop shape on the basis of two radii of its curvature $^{36}$ and is recommended for high contact angles of symmetric, high-volume drops in Appendix A.6 of the corresponding standard DIN 55660-2:2011-12.

Scanning electron microscopy (SEM) was performed on a Quanta 250 FEG microscope (FEI, Hillsboro, Oregon, USA). Samples were sputter-coated with gold and scanned in high vacuum with an acceleration voltage of $10 \mathrm{kV}$. X-ray diffraction (XRD) measurements were carried out using a SmartLab 5-Axis $\mathrm{X}$-ray diffractometer (Rigaku, Tokyo, Japan) with a $\mathrm{Cu} \mathrm{K} \alpha$ radiation (wavelength $0.154059 \mathrm{~nm}$ ) at $40 \mathrm{kV}$ and $30 \mathrm{~mA}$.

\section{Results and discussion}

\section{Properties of AKD dispersions}

The preparation parameters of dispersions together with the particle properties obtained are listed in Table 1 . Particle size was influenced by the intrinsic wax concentration and, even more clearly, by the preparation method. The use of high energy ultrasonication (dispersions V-VIII) resulted in mean particle sizes below $300 \mathrm{~nm}$. Target wax concentration of $10 \mathrm{~g} \mathrm{~L}^{-1}$ led to opaque-whitish dispersions, indicating a higher final concentration than in transparent dispersions prepared using only $1 \mathrm{~g}$ $\mathrm{L}^{-1}$ of wax. The cooling method had no influence on ultrasonicated variants. The properties of the dispersions I-IV, prepared by means of blending, showed higher deviations. Ice- 
cooling yielded smaller mean particle size and PDI than slow cooling at room temperature. Dispersion III was the only specimen with a mean particle size above $1 \mu \mathrm{m}$ and had a high DPI of 0.56. Apparently, a high number of large particles was lost during filtration.

Based on the requirement of high particle concentration in dispersion, two variants with distinctly different mean particle size were selected for further experiments. The variants IV and VII, with mean particle sizes of $841 \mathrm{~nm}$ and $291 \mathrm{~nm}$, respectively, fulfilled these requirements. In the following paragraphs, they will be referred to as "AKD-841 nm" and "AKD-291 nm", respectively.

In the production of AKD dispersions, surfactants are commonly used to provide stability. ${ }^{31}$ Such stabilizers can affect the surface energy of the dispersed particles and, consequently, lower the hydrophobisation effect achieved on a treated surface. The stability of colloidal suspensions depends on the sum of van der Waals attractive forces and electrostatic repulsive forces. ${ }^{37}$ Zeta potential provides information on the latter and is therefore often related to colloidal stability. Nanoparticle dispersions with absolute zeta potential values above $30 \mathrm{mV}$ are usually presumed highly stable. In this work, fairly stable surfactant-free AKD dispersions were obtained, which was supported by their zeta potential values. For the variant AKD-841 nm, a zeta potential value of $-31.6 \mathrm{mV}$ was measured, whereas a slightly lower (but, more importantly, higher absolute) value of $-38.1 \mathrm{mV}$ was determined for AKD-291 $\mathrm{nm}$. We did not observe any changes of the stability of dispersions within the first week after their preparation. Dispersions were used for spraying applications without any difficulty. During longer storage, some particles started to form agglomerations that floated on the surface. Still, the dispersions remained opaque and whitish, indicating a rather high remaining concentration of homogeneously dispersed particles. It is proposed that surface charge inherent to the AKD particles was responsible for the temporary stability of the dispersions.

Representative particle size distribution curves obtained by DLS measurements of both variants are shown in Fig. 2 . Samples of AKD-841 nm were mainly bidisperse. Small size particles showed an average intensity peak at approximately $170 \mathrm{~nm}$, and a larger particle peak at approximately

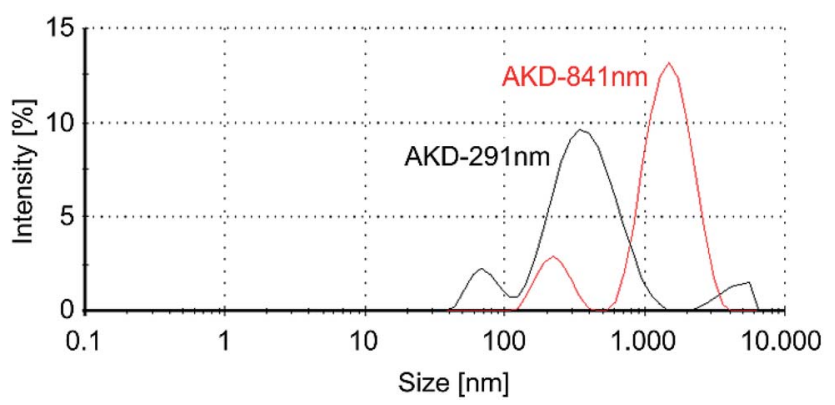

Fig. 2 Exemplary particle size distribution measurements of AKD dispersions.
$1270 \mathrm{~nm}$, with a polydispersity index (PDI) of 0.44. For AKD$291 \mathrm{~nm}$, comparable peaks were located at approximately $90 \mathrm{~nm}$ and $540 \mathrm{~nm}$ with a mean PDI of 0.42. Some measurements of that variant (such as the example shown in Fig. 2) revealed a third fraction at approximately $5 \mu \mathrm{m}$ particle size. The intensity of Rayleigh scattering, which is the underlying concept of DLS measurements, is proportional to the sixth power of the radius. ${ }^{38}$ Thus, large particles are generally overrepresented in DLS intensity measurements and these peaks correspond to a rather small number of particles. The preparation of AKD-291 nm by means of high-energy ultrasonication resulted in very small average particle size and high absolute zeta potential values. Nonetheless, the straightforward blending method used for AKD$841 \mathrm{~nm}$ also resulted in stable dispersions in spite of the higher average particle size obtained.

AFM images of particles from highly diluted and then dried dispersions confirmed bidispersity. Fig. 3a shows a high number of particles with a mean particle size of approximately $200 \mathrm{~nm}$. The majority of single particles reaches heights of only 20-50 $\mathrm{nm}$ and thus can be described as
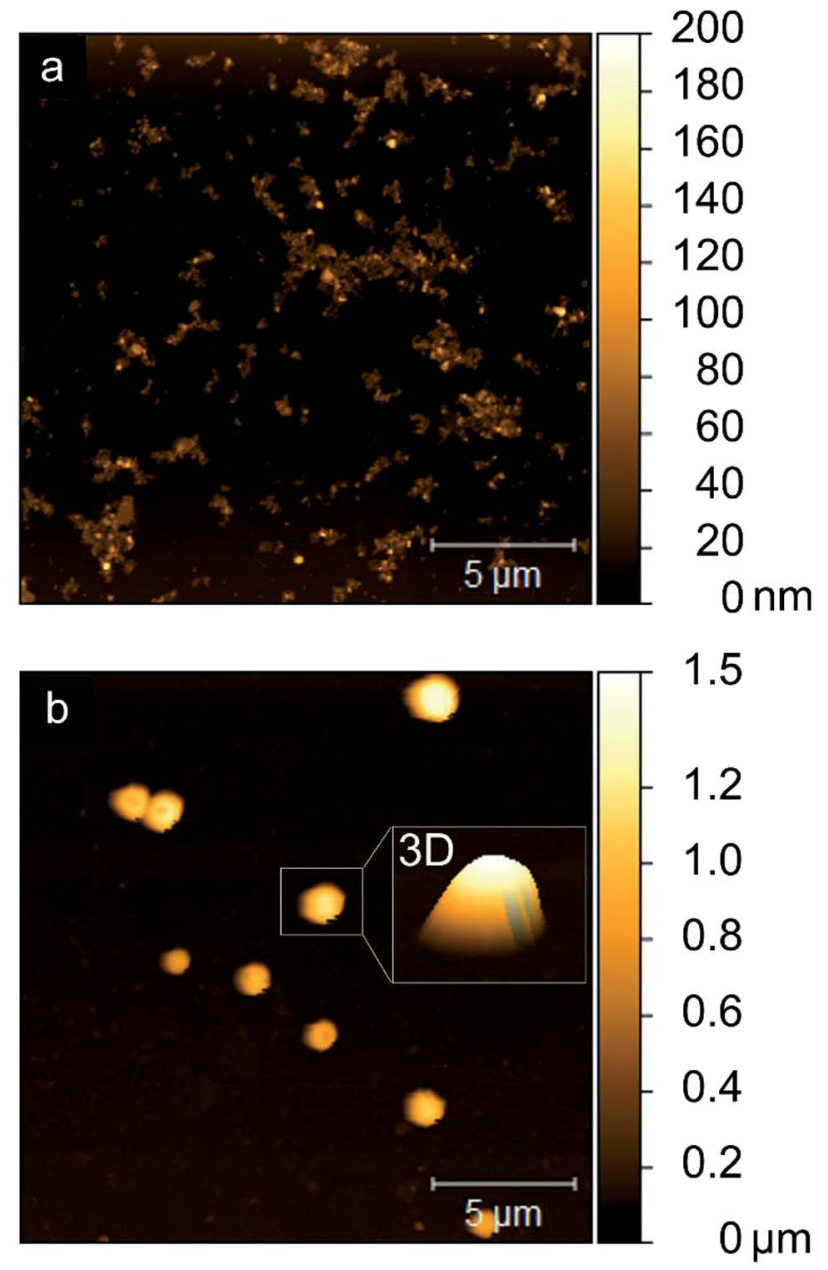

Fig. 3 AFM images of dried AKD-841 nm suspension showing a small (a) and a large particle fraction (b). The $z$-axis value scale of the 3D inset was set 1 to prevent height distortion in the depiction of the particle. 
rather flat spheroids. Fig. 3b shows particles of the same dispersion, which correspond to the second DLS intensity peak. Diameters of 1-2 $\mu \mathrm{m}$ and heights up to $1.3 \mu \mathrm{m}$ indicate a rather spherical shape, as is evident from the threedimensional depiction of a particle in the inset in Fig. $3 \mathrm{~b}$.

\section{Influence of the spray treatment on microscale roughness and optical properties of wood surfaces}

The surface characteristics of wood are determined by various factors such as the material's fibrous anatomical structure, density deviations, annual ring variation, and processing parameters such as the type of machining used. ${ }^{39}$ Consequently, sanded uncoated wood surfaces possess microscale roughness. To provide water-repellency, solid wood substrate was sprayed with AKD dispersions between 2 and 12 times. Fig. 4 shows SEM images of sanded wood that appeared comparatively even (Fig. 4a), and wood after different numbers of spraying repetitions with aqueous AKD dispersion (Fig. 4b and c). The waterbased spray treatment resulted in the swelling of fibers exposed to the surface. After four repetitions, fibers of millimeters in length were clearly protruding from the surface, increasing the surface roughness to hundreds of micrometers. Nevertheless, little influence of the deposited wax particles on roughness was visible at this level of magnification (Fig. 4b). After 10 spraying cycles, the swollen fibers appeared puttied with a layer of wax. Further, agglomerated particles with spherical or spheroidal shapes of diameters up to $150 \mu \mathrm{m}$ introduced an additional level of roughness at this high number of spraying cycles (arrows, Fig. 4c).

Increasing amounts of deposited AKD wax did not only influence the micro-level surface structure, but also the optical properties. Fig. 5 shows how color change, expressed by $\Delta E^{*}$, increased with an increasing number of spraying cycles. The corresponding photograph of the samples shows a gradual lightening from the native reddish beech wood color to a whitish surface. On variants sprayed 12 times, not only obvious color change, but even large wax agglomerations were visible. Notably, coatings obtained by means of only two

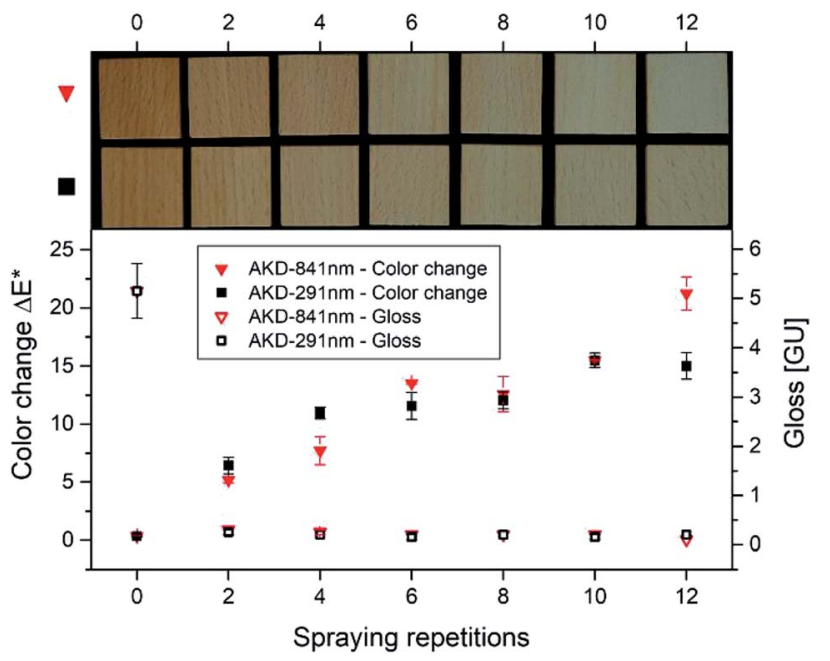

Fig. 5 Color change, gloss after the spraying treatment, and a photograph of samples sprayed 2-12 times with AKD-841 nm or AKD-291 nm dispersion. Error bars represent standard deviations.

spraying repetitions were transparent, preserving the natural appearance of such variants. The gloss values diminished from approximately $6 \mathrm{GU}$ for uncoated wood to almost $0 \mathrm{GU}$ after only two spraying repetitions, indicating a strong effect of wax coating on surface gloss. Several studies on optics have shown, that increasing surface roughness increases diffuse light scattering and therefore reduces gloss. ${ }^{\mathbf{4 0 , 4 1}}$ Hence, almost completely mat surfaces indicated distinctive surface structures after the spraying of wax dispersions.

\section{Influence of wax particle size and spraying repetitions on surface structure and wetting behavior of wood surfaces}

Previous studies have shown that water-repellency is limited even for the chemically lowest surface free energy materials, as long as insufficient surface structure is created. ${ }^{42}$ To investigate the success of our intention of equipping wood substrate with small-scale roughness by means of AKD particles, we performed
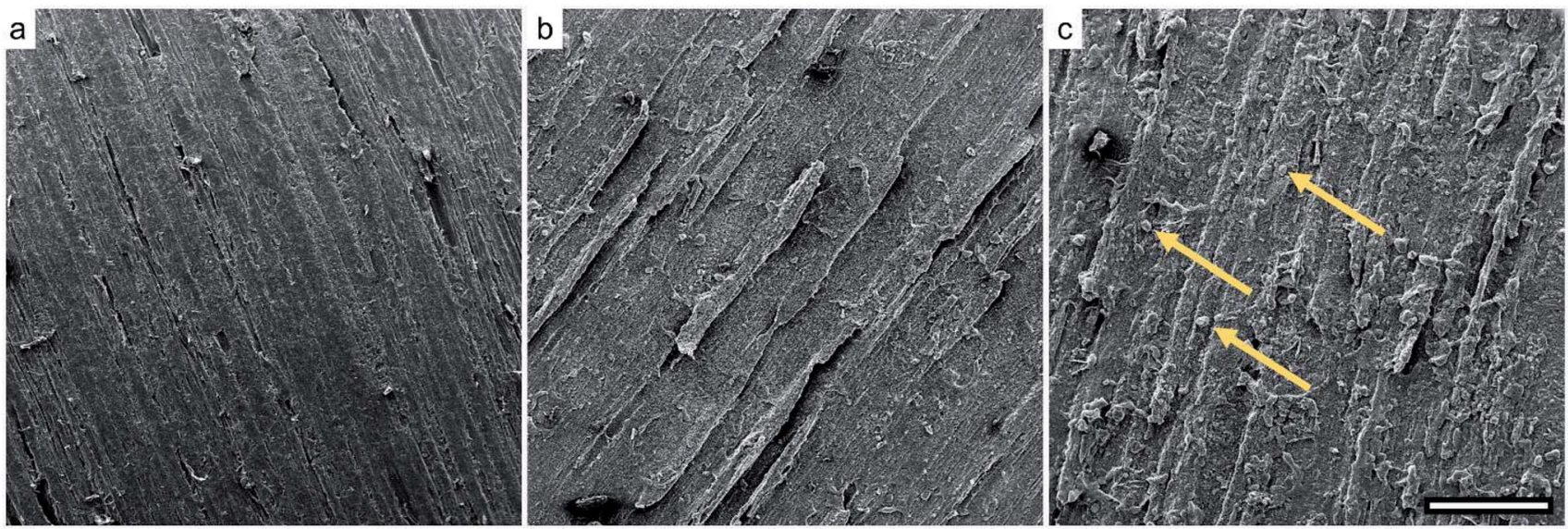

Fig. 4 SEM images of sanded wood (a) and wood sprayed $4 \times(\mathrm{b})$ and $10 \times$ (c) with AKD-291 nm reveal changes of the surface roughness during the spraying treatment. Scale bar corresponds to $500 \mu \mathrm{m}$. 
SEM and WCA measurements. Fig. 6 and 7 show surfaces of samples sprayed 2-10 times (a-e) with AKD-871 nm and AKD$291 \mathrm{~nm}$ dispersions, respectively. The SEM images revealed that sub-micron particles sprayed onto the wood surface assembled into numerous flat, upright wax platelets with lengths of about 1-5 $\mu \mathrm{m}$ and widths of approximately 100-300 $\mathrm{nm}$. Collectively, the assembled platelets form branched, porous wax layers on the substrate surfaces.

The coverage of the surface area with thin platelets is visible in Fig. 6a, but the structures of some regions seem underdeveloped when only two spraying repetitions of AKD-841 nm dispersion were performed (boxes in Fig. 6a). Small, hardly connected platelets rather than a branched and interconnected layer, and a relatively high number of spherical particles, that had not (yet) transformed into platelets (arrows, inset) are present. Four spraying repetitions (Fig. 6b) resulted in a more homogeneous surface structure, but large particles still remain in spherical shape (arrows, inset). Some small regions lack wax particles, resulting in uncovered parts or very small platelets (box). By means of six spraying repetitions, a sufficient amount of wax was deposited inducing the development of a smooth, rather homogeneous AKD coating (Fig. 6c). No obvious surface defects and a decreasing number of spheres were detected. This tendency persisted also for surfaces sprayed 8-10 times.
Consequently, hardly any large particles originating from the original dispersions are present in Fig. 6d and e. It was assumed, that the extended exposure to temperature during the iterated drying process promoted the transformation from large wax spheres into thin platelets on repeatedly sprayed substrates. Excessively deposited AKD formed comparatively large wax agglomerations in the diameter range of tens of micrometers (box Fig. 6e), already visible in Fig. 4c.

The wetting behavior of the coated wood substrates is shown in Fig. 6f, expressed by mean WCA measured 1 min after droplet deposition. Overall, the initially hydrophilic surface of beech wood was endowed with excellent water-repellency. A mean WCA of approximately $139^{\circ}$ was measured after only two spraying repetitions with AKD-841 nm. Remarkably, even the imperfect surface structure of this variant resulted in an incipient WCA of $152^{\circ}$ but values decreased over the total observation time. All other samples reached a superhydrophobic state expressed by WCA ranging from approximately $158-162^{\circ}$. These contact angles remained constant throughout the full measurement period, as depicted in the insert graph of a variant sprayed four times. An exemplary image extracted from the automatic WCA calculation is also shown in Fig. 6f, demonstrating the accuracy of the Young-Laplace fit for modelling a large volume droplet shape.
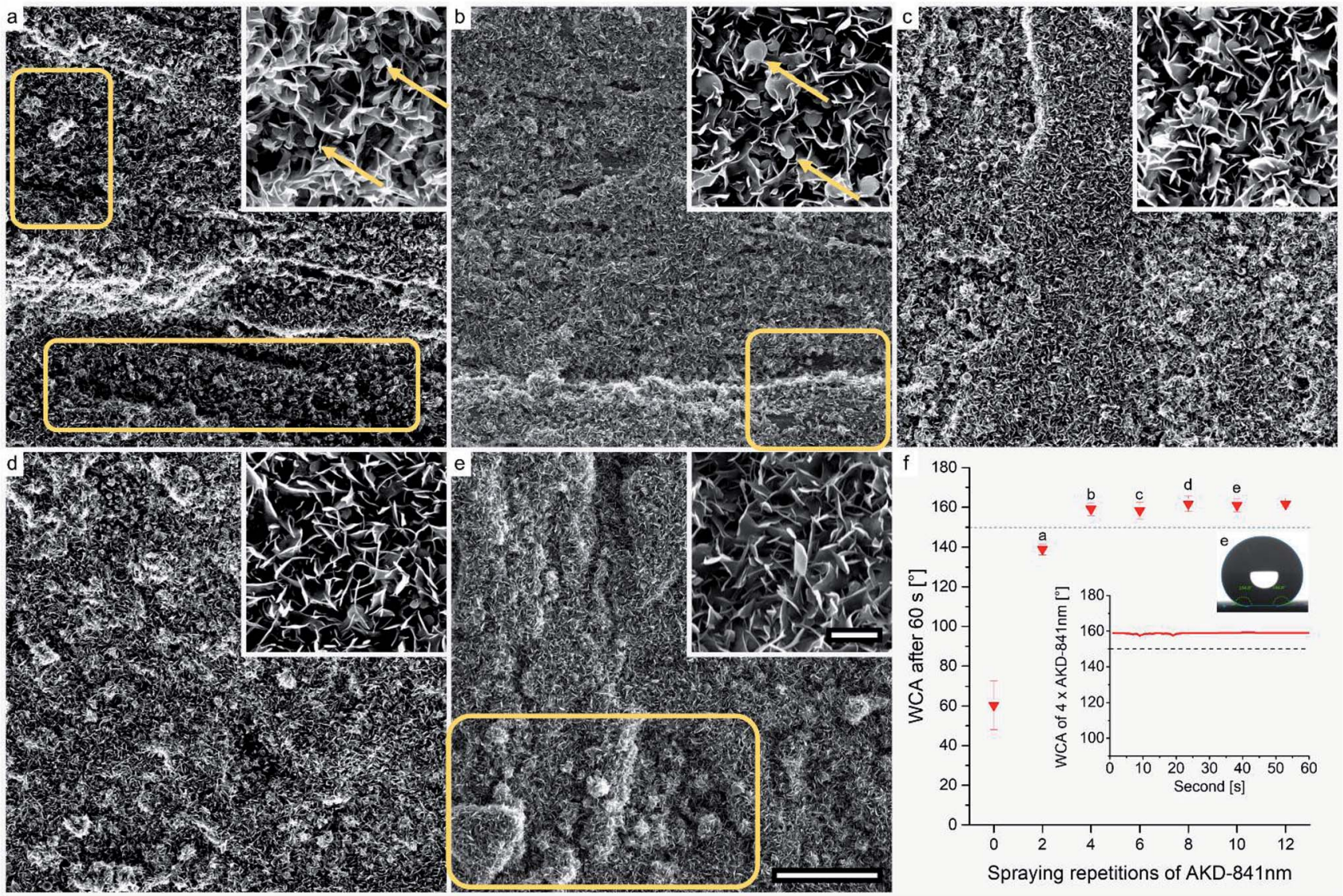

Fig. 6 SEM images of samples sprayed 2-10 times with AKD-841 nm, scale bars correspond to $50 \mu \mathrm{m}$ and $5 \mu \mathrm{m}$ (inset), respectively (a-e). Influence of spraying repetitions on the WCA after $60 \mathrm{~s}$ of exposure, exemplary WCA behavior over $60 \mathrm{~s}$, and an image of the maximum WCA measurement of a sample sprayed $10 \times$. Error bars represent standard deviation (f). 

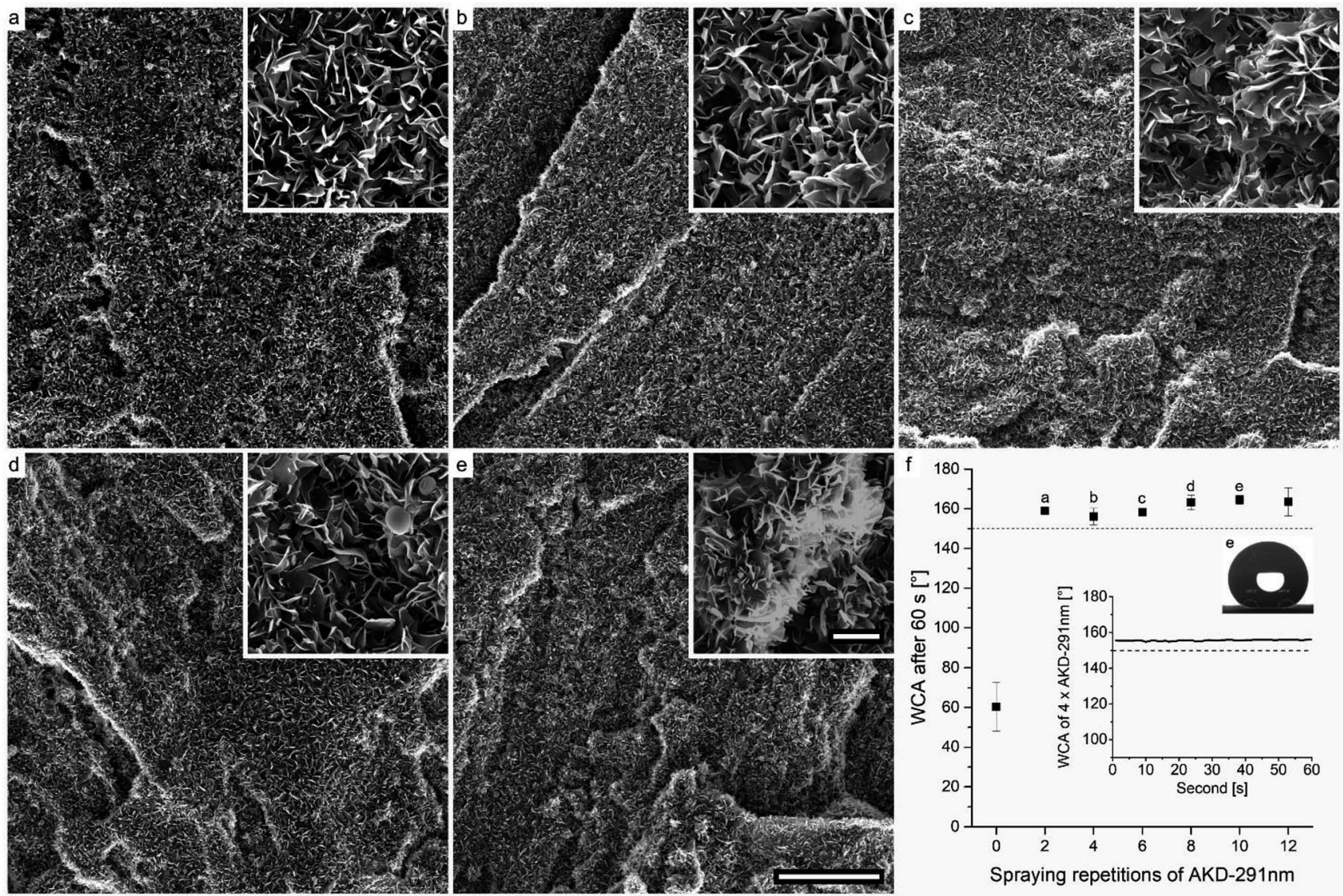

Fig. 7 SEM images of samples sprayed 2-10 times with AKD-291 nm, scale bars correspond to $50 \mu \mathrm{m}$ and $5 \mu \mathrm{m}$ (inset), respectively (a-e). Influence of spraying repetitions on the WCA after $60 \mathrm{~s}$ of exposure, exemplary behavior over $60 \mathrm{~s}$, and an image of the maximum WCA measurement of a sample sprayed $10 \times$. Error bars represent standard deviation (f).

Coatings prepared using the AKD-291 nm variant are displayed in Fig. 7. They reveal a similar wax layer as observed for the AKD-841 nm dispersion (Fig. 6). Interestingly, little influence of the intrinsic particle size on the resulting shape of single platelets was observed. Even so, it appears that the usage of smaller particles favors a more homogeneous coverage of the surface already at a low number of spraying repetitions (Fig. 7a and $b$ ).

Considerable formation of large agglomerations due to excess AKD was observed after the sixth spraying repetition (Fig. 7c). The inset of Fig. 7e shows that such wax agglomerations are covered with a layer of thin platelets. Overall, few large spherical shapes were found. Same as with AKD-841 nm the sophisticated surface structure lead to stable superhydrophobic behavior for all AKD-291 nm variants, starting with as few as two spraying-drying cycles. The mean WCA ranged from approximately $156-165^{\circ}$ for variants sprayed four and ten times, respectively. Apparently, for both dispersions used there existed a critical number of spraying repetitions necessary to reach superhydrophobic water-repellency. For AKD-841 nm it took four iterations, whereas a stable WCA of $159^{\circ}$ was already after spraying twice with AKD-291 nm. Any additional deposition of wax did not seem to have significant influence on the wetting behavior.

\section{Influence of drying conditions on surface structure and} wetting behavior

DSC measurements of AKD revealed a specific melting behavior shown in Fig. 8. Considering the melt range shown, different samples of one variant $(4 \times$ AKD-291 nm) were repeatedly sprayed and dried for $5 \mathrm{~min}$ at temperatures from $20-80{ }^{\circ} \mathrm{C}$ in order to investigate the impact of the drying temperature on surface morphology and wettability.

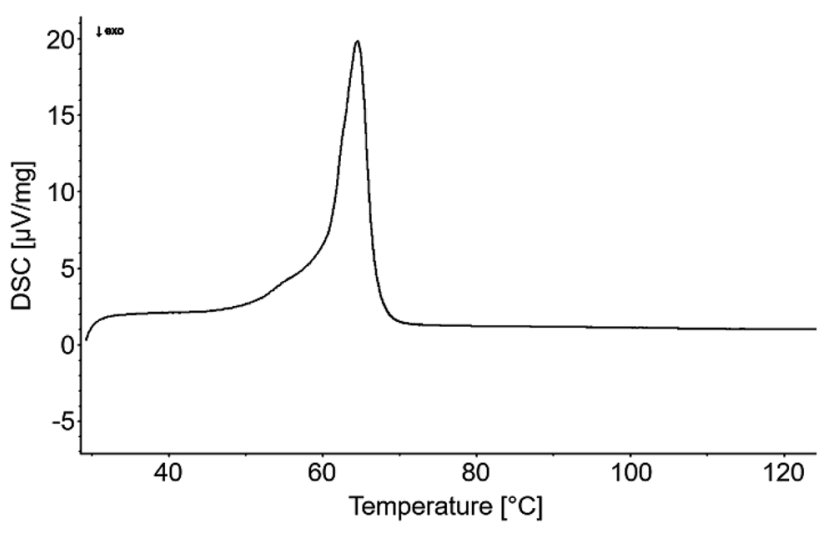

Fig. 8 Differential scanning calorimetry of AKD giving insight on the material's melting range. 
SEM images (Fig. 9) reveal wax platelets which are not as well-developed as the ones present on surfaces shown in Fig. 6 and 7. Since the variants of Fig. 9 were dried for a shorter period ( 5 instead of $10 \mathrm{~min}$ ) and stored for a shorter time before the SEM investigation (3 days instead of 1 week), it is assumed that a longer drying time favors platelet growth. Nonetheless, WCA of the samples dried between $20-50{ }^{\circ} \mathrm{C}$ for only $5 \mathrm{~min}$ are clearly found in the superhydrophobic region (Fig. 9h). A slight increase of water-repellency with increasing drying temperature was observed. From a comparison of the insets in Fig. 9a-d it can be inferred that increasing temperature favors self- assembly, resulting in a more distinctive surface structure. A drying temperature of $60{ }^{\circ} \mathrm{C}$, located at the onset of the materials melting range, resulted in partial melting and therefore increased distance between the remaining platelets. WCA was slightly reduced but still in the superhydrophobic range. Drying at $70{ }^{\circ} \mathrm{C}$, a temperature beyond the DSC-derived melting range, led to full flattening of most surface parts. Only few single platelets of small size remained on the surface after exposure to $80{ }^{\circ} \mathrm{C}$. Comparatively large wax agglomerations in the microrange but little remaining sub-micro roughness resulted in a mean WCA of $137^{\circ}$.
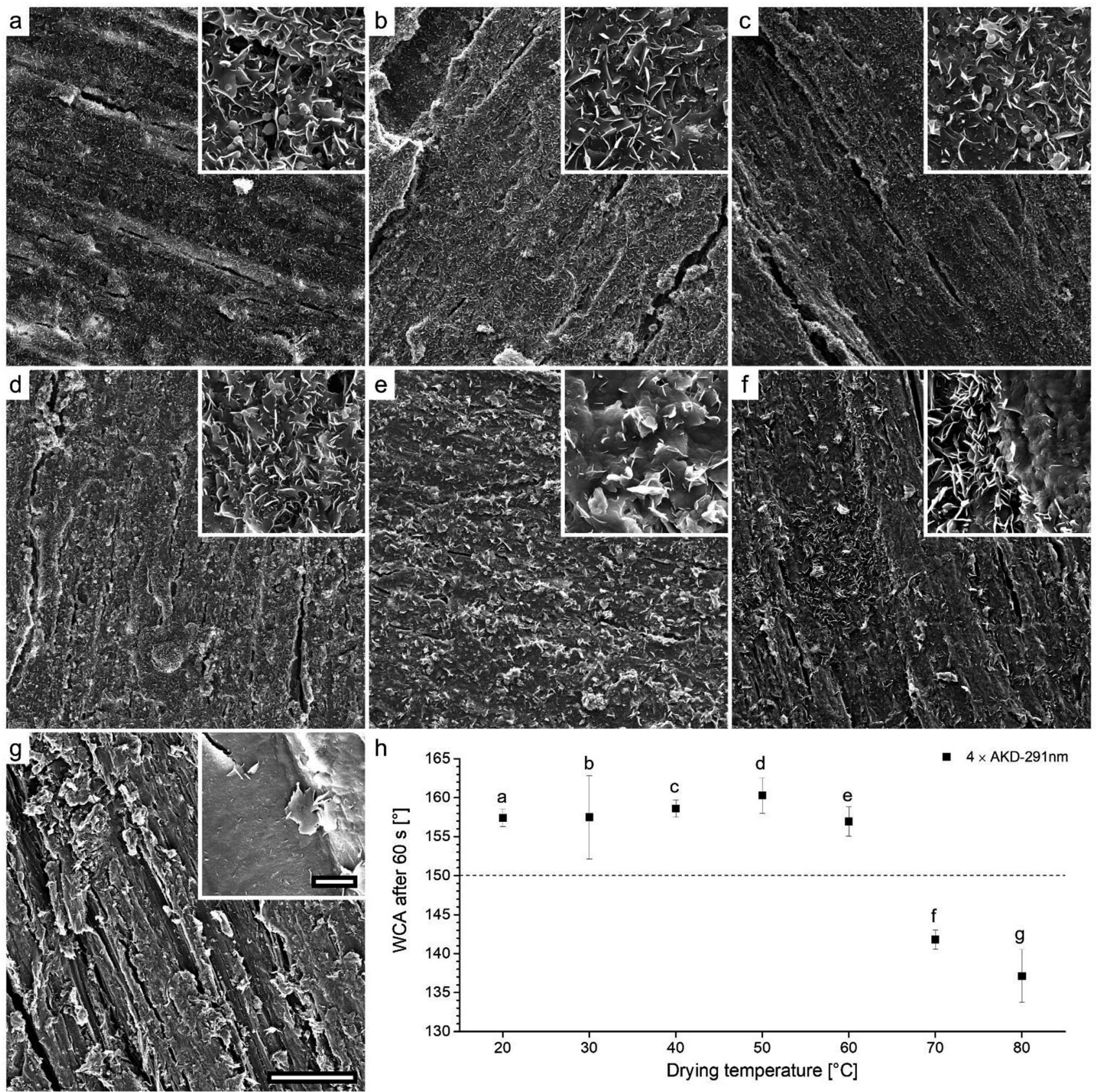

h

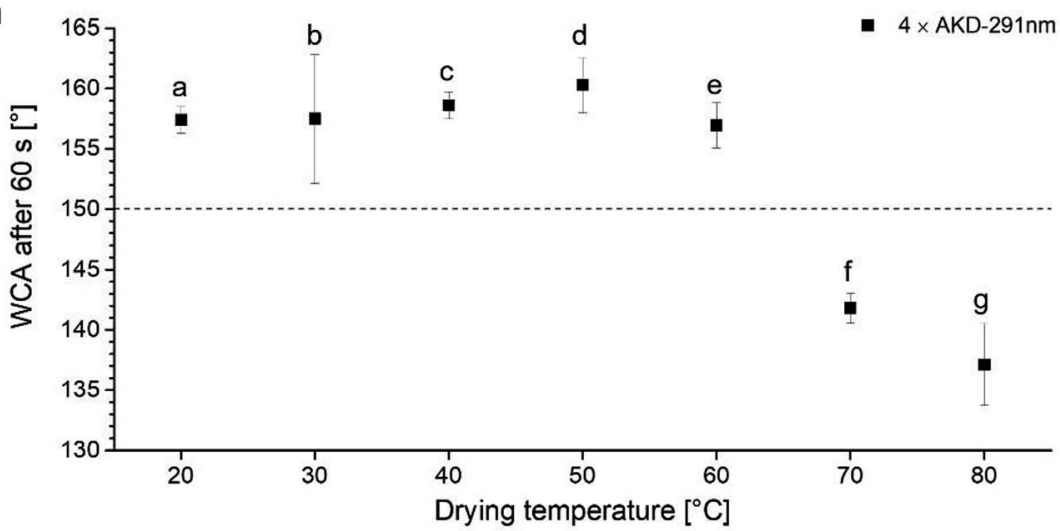

Fig. 9 SEM images of samples sprayed $4 \times$ with AKD-291 nm and dried at $20-80{ }^{\circ} \mathrm{C}$, scale bars correspond to $50 \mu \mathrm{m}$ and $5 \mu \mathrm{m}$ (inset), respectively $(\mathrm{a}-\mathrm{g})$. Influence of drying temperature on the WCA after $60 \mathrm{~s}$ of exposure, error bars represent standard deviation (h). 


\section{Surface curing}

A follow-up experiment to investigate effects of the time of exposure to temperature was carried out using a variant treated with four spraying cycles of AKD-291 nm. The variant was iteratively dried at $40{ }^{\circ} \mathrm{C}$, kept in the oven for $24 \mathrm{~h}$ at the same temperature and thereafter stored for two days at $20{ }^{\circ} \mathrm{C}$. SEM images presented in Fig. 10 revealed a very homogeneous, welldeveloped porous wax layer. No trace of large, spherical particles as was the case in earlier experiments applying shorter drying times (Fig. 6, 7, and 9) was observed. The WCA for this specimen was $166.15^{\circ}$, which is the highest value achieved within the present study.

\section{Structure of crystalline platelets}

Upright platelets appear to be very efficient structures in terms of water repellence. Over the course of evolution, nature developed geometries very similar to the ones observed for AKD in the present study. The carnivorous pitcher plant (Nepentes alata) arranges crystalline wax platelets rectangular to the apparent surface to create superhydrophobic surfaces. ${ }^{\mathbf{4 3 , 4 4}}$ While different in terms of surface coverage and their degree of development from originally spherical particles, the AKD platelets observed in the present study were of fairly uniform geometry (lengths of about 1-5 $\mu \mathrm{m}$ and widths of approximately 100-300 nm). AKD structures on superhydrophobic paper surfaces ${ }^{35}$ exhibited highly similar geometry, although a completely different process, i.e. the rapid expansion of supercritical $\mathrm{CO}_{2}$, was used. Since it is well known that crystalline regions may develop in AKD under certain conditions, ${ }^{45} \mathrm{X}$-ray diffraction measurements

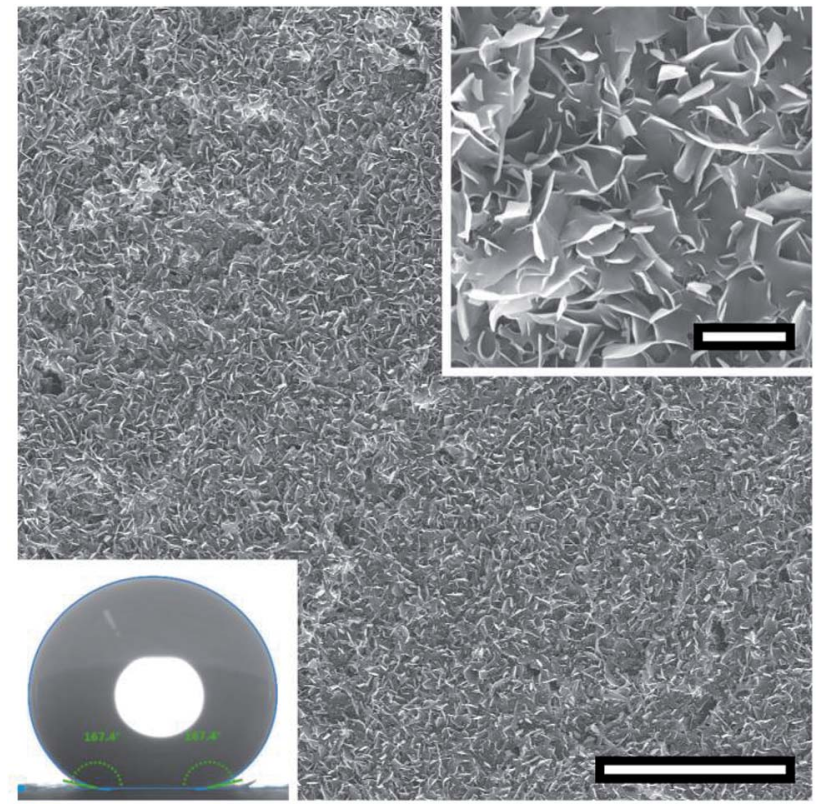

Fig. 10 SEM images of a sample sprayed $4 \times$ with AKD-291 nm, dried and then kept at $40{ }^{\circ} \mathrm{C}$. Scale bars correspond to $50 \mu \mathrm{m}$ and $5 \mu \mathrm{m}$ (inset), respectively. Bottom inset: Image of the maximum WCA measurement.

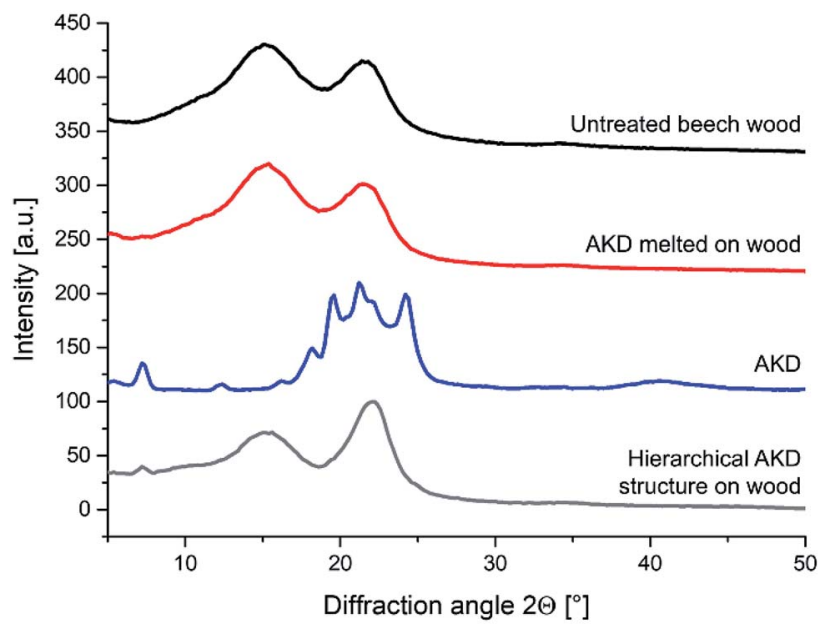

Fig. $11 X$-ray diffractograms of untreated beech wood, melted AKD on wood, pure AKD, and hierarchical AKD structure (platelets) on wood.

were carried out in order to obtain additional information on the molecular structure of AKD platelets. An X-ray diffractogram of pure AKD grain melted at $103{ }^{\circ} \mathrm{C}$ and then resolidified shows a characteristic diffraction pattern in the range of $2 \theta 15-27^{\circ}$ (Fig. 11). This pattern is overlapping with the typical cellulose peak detected on measured wood samples and therefore was difficult to evaluate. ${ }^{46}$ Furthermore, a typical diffraction peak at around $2 \theta 7^{\circ}$ arises in a region where no cellulose signal is observed. According to literature, this peak is the result of structures formed due to secondary interactions between alkenyl chains of AKD and thus indicative of crystallinity. ${ }^{45}$ When $\mathrm{AKD}$ is applied to wood in molten state and then re-solidifies, no diffraction peak indicative of crystalline AKD structures is observed. By contrast, a specimen treated with aqueous AKD dispersion exhibiting surface structure as depicted in Fig. 6, 7, and 9, shows a fairly distinct AKD diffraction peak at $2 \theta 7^{\circ}$. It may thus be assumed that the specific geometry of AKD platelets is pre-determined by the formation of crystalline structures in AKD.

\section{Conclusion}

It was shown that surfactant-free dispersions of sub-micron AKD particles in water, which remain stable for at least one week, can be produced in a simple fashion. Upon application to wood surfaces and subsequent drying, AKD particles selfassemble to nanometer-thin platelets which endow the treated surface with superhydrophobicity. Up to the melting range, increased drying temperature favors platelet development, same as prolonged drying time.

With the exception of reduced surface gloss, no unwanted alterations in the optical appearance of treated wood surfaces were observed, making the method a promising approach for facile and cost-effective hydrophobisation of woody substrates. 


\section{Funding}

Financial support by the Austrian COMET program (COMET K1 centre WOOD, project number 865905) is gratefully acknowledged. The funding sources had no involvement in study design, in the collection, analysis and interpretation of data, in the writing of the report, or in the decision to submit the article for publication.

\section{Standards}

DIN EN ISO 2813:2015-02 Paints and varnishes - Determination of gloss value at $20^{\circ}, 60^{\circ}$ and $85^{\circ}$ (ISO 2813:2014); German version EN ISO 2813:2014.

DIN 55660-2:2011-12 Paints and varnishes - Wettability Part 2: Determination of the free surface energy of solid surfaces by measuring the contact angle.

\section{Conflicts of interest}

There are no conflicts to declare.

\section{Acknowledgements}

Elisabeth Lackinger and Kemira Chemie GesmbH in Krems, Austria, are acknowledged for providing AKD. Daniela Keckesova and Julien Jaxel are acknowledged for performing XRD and DSC measurements, respectively.

\section{References}

1 M. F. Ashby and D. Cebon, Le Journal de Physique IV, 1993, 3, C7.

2 R. J. Ross, USDA Forest Service, Forest Products Laboratory, General Technical Report FPL-GTR-190, 2010: vol. 1, p. 509.

3 Food and Agriculture Organization of the United Nations (FAO), FAOSTAT data on Forestry Production and Trade, Total worldwide sawnwood production quantity, http:// www.fao.org/faostat/en/\#data/ (Regions: World + (total), Elements: Production quantity, Items aggregated: sawnwood + (total), Years: all; accessed 23 April 2019).

4 C. A. Hill, Wood modification: chemical, thermal and other processes, John Wiley \& Sons, 2007.

5 R. M. Rowell, Wood Mater. Sci. Eng., 2006, 1, 29-33.

6 B. Esteves and H. Pereira, BioResources, 2008, 4, 370-404.

7 D. Sandberg, A. Kutnar and G. Mantanis, iForest Biogeosciences and Forestry, 2017, 10, 895-908.

8 Z. Guo and F. Yang, Surfaces and Interfaces of Biomimetic Superhydrophobic Materials, John Wiley \& Sons, 2017.

9 L. Cao, A. K. Jones, V. K. Sikka, J. Wu and D. Gao, Langmuir, 2009, 25, 12444-12448.

10 L. Feng, S. Li, Y. Li, H. Li, L. Zhang, J. Zhai, Y. Song, B. Liu, L. Jiang and D. Zhu, Adv. Mater., 2002, 14, 1857-1860.

11 W. Barthlott and C. Neinhuis, Planta, 1997, 202, 1-8.

12 X.-M. Li, D. Reinhoudt and M. Crego-Calama, Chem. Soc. Rev., 2007, 36, 1350-1368.

13 B. Bhushan and Y. C. Jung, Prog. Mater. Sci., 2011, 56, 1-108.
14 C. Wang, C. Piao and C. Lucas, J. Appl. Polym. Sci., 2011, 119, 1667-1672.

15 S. Wang, J. Shi, C. Liu, C. Xie and C. Wang, Appl. Surf. Sci., 2011, 257, 9362-9365.

16 S. Wang, C. Liu, G. Liu, M. Zhang, J. Li and C. Wang, Appl. Surf. Sci., 2011, 258, 806-810.

17 F. Liu, S. Wang, M. Zhang, M. Ma, C. Wang and J. Li, Appl. Surf. Sci., 2013, 280, 686-692.

18 S. Jia, M. Liu, Y. Wu, S. Luo, Y. Qing and H. Chen, Appl. Surf. Sci., 2016, 386, 115-124.

19 L. Gao, Y. Lu, X. Zhan, J. Li and Q. Sun, Surf. Coat. Technol., 2015, 262, 33-39.

20 Y. Qing, M. Liu, Y. Wu, S. Jia, S. Wang and X. Li, Results Phys., 2017, 7, 1705-1711.

21 B. Hui, D. Wu, Q. Huang, L. Cai, G. Li, J. Li and G. Zhao, RSC Adv., 2015, 5, 73566-73574.

22 H. Guo, P. Fuchs, K. Casdorff, B. Michen, M. Chanana, H. Hagendorfer, Y. E. Romanyuk and I. Burgert, Adv. Mater. Interfaces, 2017, 4, 1600289.

23 S. M. Shah, U. Zulfiqar, S. Z. Hussain, I. Ahmad, I. Hussain and T. Subhani, Mater. Lett., 2017, 17-20.

24 Y. Chen, H. Wang, Q. Yao, B. Fan, C. Wang, Y. Xiong, C. Jin and Q. Sun, J. Mater. Sci., 2017, 52, 7428-7438.

25 K. Tu, L. Kong, X. Wang and J. Liu, Holzforschung, 2016, 70, 1039-1045.

26 C. Liu, S. Wang, J. Shi and C. Wang, Appl. Surf. Sci., 2011, 258, 761-765.

27 M. Liu, Y. Qing, Y. Wu, J. Liang and S. Luo, Appl. Surf. Sci., 2015, 330, 332-338.

28 A. Lozhechnikova, H. Bellanger, B. Michen, I. Burgert and M. Österberg, Appl. Surf. Sci., 2017, 396, 1273-1281.

29 W. Downey, US Pat., US2627477A, 1949.

30 J. C. Roberts, The chemistry of paper, Royal Society of Chemistry, 2007.

31 T. Lindström and P. T. Larsson, Nord. Pulp Pap. Res. J., 2008, 23, 202-209.

32 P. Samyn, J. Mater. Sci., 2013, 48, 6455-6498.

33 S. Shibuichi, T. Onda, N. Satoh and K. Tsujii, J. Phys. Chem., 1996, 100, 19512-19517.

34 T. Onda, S. Shibuichi, N. Satoh and K. Tsujii, Langmuir, 1996, 12, 2125-2127.

35 C. Quan, O. Werner, L. Wågberg and C. Turner, J. Supercrit. Fluids, 2009, 49, 117-124.

36 M. Taylor, A. J. Urquhart, M. Zelzer, M. C. Davies and M. R. Alexander, Langmuir, 2007, 23, 6875-6878.

37 S. Bhattacharjee, J. Controlled Release, 2016, 235, 337-351.

38 C. Barnett, J. Phys. Chem., 1942, 46, 69-75.

39 M. Kilic, S. Hiziroglu and E. Burdurlu, Building and Environment, 2006, 41, 1074-1078.

40 J. Järnström, P. Ihalainen, K. Backfolk and J. Peltonen, Appl. Surf. Sci., 2008, 254, 5741-5749.

41 H. Assender, V. Bliznyuk and K. Porfyrakis, Science, 2002, 297, 973-976.

42 T. Nishino, M. Meguro, K. Nakamae, M. Matsushita and Y. Ueda, Langmuir, 1999, 15, 4321-4323. 
43 I. Scholz, M. Bückins, L. Dolge, T. Erlinghagen, A. Weth, F. Hischen, J. Mayer, S. Hoffmann, M. Riederer and M. Riedel, J. Exp. Biol., 2010, 213, 1115-1125.

44 L. Wang and Q. Zhou, Sci. Rep., 2016, 6, 19907.
45 Y. Yoshida and A. Isogai, Cellulose, 2006, 13, 637-645.

46 Y. Yan, H. Amer, T. Rosenau, C. Zollfrank, J. Dörrstein, C. Jobst, T. Zimmermann, J. Keckes, S. Veigel, W. GindlAltmutter and J. Li, Cellulose, 2016, 23, 1189-1197. 